\title{
Elastic Domains in Antiferromagnets on Substrates
}

\author{
Efim A. Brener ${ }^{1}$ and V. I. Marchenko ${ }^{2}$ \\ ${ }^{1}$ Institut für Festkörperforschung, Forschungszentrum Jülich, D-52425 Jülich, Germany \\ ${ }^{2}$ P. L. Kapitza Institute for Physical Problems, RAS 119334, Kosygina 2, Moscow, Russia
}

(Received 9 March 2006; published 10 August 2006)

\begin{abstract}
We consider periodic domain structures which appear due to the magnetoelastic interaction if the antiferromagnetic crystal is attached to an elastic substrate. The peculiar behavior of such structures in an external magnetic field is discussed. In particular, we find the magnetic field dependence of the equilibrium period and the concentrations of different domains.
\end{abstract}

DOI: 10.1103/PhysRevLett.97.067204

PACS numbers: 75.60.Ch, 74.72.Bk, 75.25.+z

Spontaneous breaking of any discrete rotational symmetry in monocrystals leads to the appearance of degenerate phases characterized by different orientations of the order parameter. The phase boundaries have a positive (free) energy; otherwise, the system would be thermodynamically unstable. Thus, in equilibrium one should observe a single phase. This is, however, not the case for the phases with spontaneous magnetization (or electrical polarization). Periodic domain structures are formed in such materials in order to reduce the large, proportional to the volume, energy of the magnetic (or electric) field.

Using the electron spin resonance technique, Janossy et al. [1,2] demonstrated the existence of the equilibrium domain structure in antiferromagnetic $\mathrm{YBa}_{2} \mathrm{Cu}_{3} \mathrm{O}_{6+x}$ and in $\mathrm{Y}_{1-x} \mathrm{Ca}_{x} \mathrm{Ba}_{2} \mathrm{Cu}_{3} \mathrm{O}_{6}$. The domain structure was easily modified by the external magnetic field and restored after switching the field off. The resonance method does not provide information about characteristic sizes and the arrangement of the domains. Recently, Vinnikov et al. [3] directly observed periodic domain structures in antiferromagnetic $\mathrm{TbNi}_{2} \mathrm{~B}_{2} \mathrm{C}$ by the "finest magnetic-particle decoration technique" [4]. Neither spontaneous magnetization nor electrical polarization is seen in this material. Nevertheless, regular and reversible (by the change of the temperature and magnetic field) domain structures are realized with a periodicity of the order of a few microns in the samples of plate shape with a thickness $0.5 \mathrm{~mm}$.

In this Letter, we discuss a possible scenario for the appearance of such periodic domain structures and the behavior of the structures in an external magnetic field. If a monocrystal is attached to some elastic substrate, the domain structure should inevitably arise in any orientational phase transition in order to minimize the strain energy. Indeed, the stress free (but not strain free) monodomain state is realized if we discuss free surface boundary conditions. If the monodomain crystal is attached to some elastic substrate, stresses arise, and we lose a large, proportional to the crystal volume, energy. In this case, the appearance of the domain structure, with a period much smaller than the crystal size, will drastically diminish the energy of elastic deformations.
All materials studied in the experiments of Refs. [1-3] are collinear easy plane antiferromagnets with tetragonal crystal symmetry. For definiteness' sake, we discuss this case. For the description of the antiferromagnetic states, we use the exchange approximation [5], treating the effects of the magnetic anisotropy, magnetoelastic effects, and the external magnetic field as perturbations. By a proper choice of the orientation of the coordinate system (say, the $x$ axis can be oriented along the [110] or [100] direction), we can write the anisotropy energy in the easy $x y$ plane as

$$
F_{\text {an }}=-\beta l_{x}^{2} l_{y}^{2},
$$

with a positive constant $\beta$; here $l_{x}$ and $l_{y}$ are the components of the antiferromagnetic unit vector $l$. The four states $l_{x}^{2}=l_{y}^{2}=1 / 2$ differ by the signs of the $x, y$ components $(\nearrow, \searrow, \swarrow, \backslash)$ and correspond to the minimum of the energy [Eq. (1)].

The elastic strain arises in the crystal due to the magnetoelastic interaction

$$
F_{\mathrm{me}}=\gamma_{1} l_{x} l_{y} u_{x y}+\gamma_{2}\left(l_{x}^{2}-l_{y}^{2}\right)\left(u_{x x}-u_{y y}\right),
$$

where $u_{i k}$ are the components of the strain tensor. For simplicity, we consider the magnetoelastic effects to be small compared to the anisotropy [Eq. (1)]. The second term in Eq. (2) is zero for the discussed states with $l_{x}^{2}=l_{y}^{2}$. The elastic energy of tetragonal crystals can be written as a sum of 6 invariants:

$$
\begin{aligned}
F_{\mathrm{el}}= & \mu_{1} u_{x y}^{2}+\mu_{2}\left(u_{x z}^{2}+u_{y z}^{2}\right)+\mu_{3}\left(u_{x x}-u_{y y}\right)^{2}+\mu_{4} u_{z z}^{2} \\
& +\mu_{5} u_{z z}\left(u_{x x}+u_{y y}\right)+\mu_{6}\left(u_{x x}+u_{y y}\right)^{2} .
\end{aligned}
$$

Minimizing the sum of the energies [Eqs. (2) and (3)], we find that, in the monodomain state of the unstressed crystal, the only nonzero component of the strain tensor is

$$
u_{x y}^{0}=\frac{\gamma_{1}}{2 \mu_{1}} l_{x} l_{y}= \pm \frac{\gamma_{1}}{4 \mu_{1}} .
$$

Consider the antiferromagnetic crystal, of plate shape, attached to a flat elastic substrate. Let the main axis of the crystal be normal to the plate. The domain structure that 
appears in this case is schematically presented in Fig. 1. The state of each domain far from the crystal-substrate boundary is one of the unstressed ground states. Additional energetic contributions arise from (i) the energy of the domain walls and (ii) the elastic energy localized near the crystal-substrate boundary in a layer of thickness of the order of the domain structure periodicity $d$. Minimization of these two contributions with respect to $d$ leads to the equilibrium period of the domain structure. This argumentation is close in spirit to arguments of Ref. [6] (see also [79]). The elastic interaction between domains and point defects was discussed in Ref. [10]. However, we stress that the additional elastic energy term appears only near the crystal-substrate boundary and does not appear near the free surface. This is a crucial difference between these elastic domains and the domains in ferromagnets.

In accordance with the symmetry of the domain structure (Fig. 1), the displacement vector $\mathbf{u}$ has only one component $u_{y}$ inside the crystal, as well as in the substrate. Therefore, the elasticity equation in the crystal reduces to

$$
\mu_{1} \partial_{x}^{2} u_{y}+\mu_{2} \partial_{z}^{2} u_{y}=0 .
$$

We assume that the substrate is elastically isotropic: $\mu_{1}=$ $\mu_{2}=2 \mu$, where $\mu$ is the shear modulus of the substrate. The boundary conditions at the sample-substrate contact surface are

$$
\left.u_{y}\right|_{z=+0}=\left.u_{y}\right|_{z=-0},\left.\quad \mu_{2} \partial_{z} u_{y}\right|_{z=+0}=\left.2 \mu \partial_{z} u_{y}\right|_{z=-0} .
$$

The displacement field inside the crystal, far from the crystal-substrate boundary, is a zigzag function $u^{0}$ as schematically presented in Fig. 2. Its expansion in Fourier series is

$$
u^{0}(x)=\sum_{n=0}^{\infty} u_{n}^{0} \sin \frac{2 \pi(2 n+1) x}{d}
$$

with coefficients

$$
u_{n}^{0}=\frac{(-1)^{n} \gamma_{1} d}{\pi^{2} \mu_{1}(2 n+1)^{2}} .
$$

It is convenient to introduce a new field $u^{+}$by the relation

$$
u_{y}=u^{0}(x)+u_{y}^{+}(x, z) .
$$

The Fourier series for $u_{y}^{+}$, in accordance with Eq. (5), is

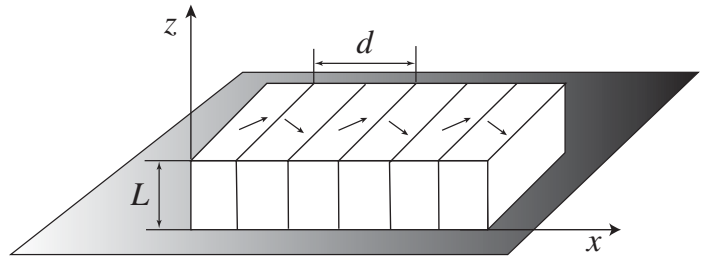

FIG. 1. Schematic presentation of the domain structure in the antiferromagnetic crystal attached to an elastic substrate.

$$
u_{y}^{+}=\sum_{n=0}^{\infty} u_{n}^{+} \sin \frac{2 \pi(2 n+1) x}{d} \exp \left\{-\frac{2 \pi(2 n+1) \sqrt{\mu_{1}} z}{\sqrt{\mu_{2}} d}\right\}
$$

The Fourier series for $u_{y}=u_{y}^{-}$in the substrate is

$$
u_{y}^{-}=\sum_{n=0}^{\infty} u_{n}^{-} \sin \frac{2 \pi(2 n+1) x}{d} \exp \left\{\frac{2 \pi(2 n+1) z}{d}\right\} .
$$

From the boundary conditions [Eq. (6)], we find

$$
u_{n}^{+}=-\frac{2 \mu u_{n}^{0}}{2 \mu+\sqrt{\mu_{1} \mu_{2}}} ; \quad u_{n}^{-}=\frac{\sqrt{\mu_{1} \mu_{2}} u_{n}^{0}}{2 \mu+\sqrt{\mu_{1} \mu_{2}}} .
$$

Now we can determine the total energy of the system per unit area in the $x y$ plane

$$
\mathcal{F}=-\mu_{1}\left(u_{x y}^{0}\right)^{2} L+\nu \frac{\mu \sqrt{\mu_{1} \mu_{2}}}{2 \mu+\sqrt{\mu_{1} \mu_{2}}}\left(u_{x y}^{0}\right)^{2} d+\frac{\sigma_{0} L}{d},
$$

where $\sigma_{0}$ is the domain wall energy, and

$$
\nu=\frac{8}{\pi^{3}} \sum_{n=0}^{\infty} \frac{1}{(2 n+1)^{3}} \approx 0.27 .
$$

The first term in Eq. (10) represents the energy gain due to the arising of the domain structure. In the monodomain state of the sample with the free surface, this is the only term of the magnetoelastic relaxation. The second term is the elastic energy due to the inhomogeneous strain near the crystal-substrate boundary. The third term accounts for the domain walls energy. Minimization of the energy [Eq. (10)] with respect to $d$ gives the equilibrium period

$$
d=d_{0}=\left(\frac{\left(2 \mu+\sqrt{\mu_{1} \mu_{2}}\right) \sigma_{0} L}{\nu \mu \sqrt{\mu_{1} \bar{\mu}_{2}}\left(u_{x y}^{0}\right)^{2}}\right)^{1 / 2} .
$$

The classical law $d \propto L^{1 / 2}$, in the limit $d \ll L$, is the crucial point for the experimental verification of the domain structure theory. In the case of antiferromagnetic domains, there exists an additional valuable experimental possibility, namely, to study the behavior of the structure in the external magnetic field.

The magnetic field changes the orientation of the antiferromagnetic vector. The corresponding term in the energy is

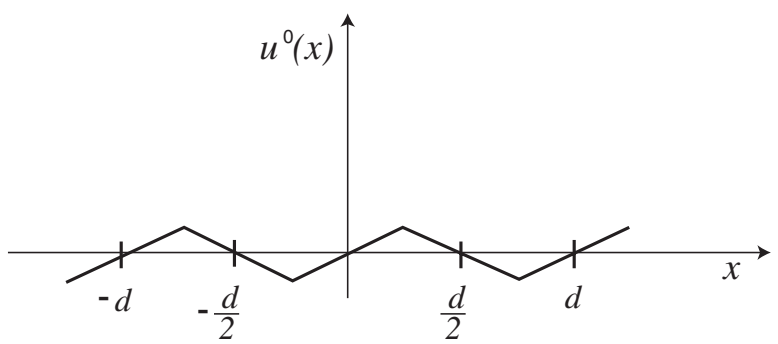

FIG. 2. The displacement field inside the crystal far from the crystal-substrate boundary. 


$$
F_{H}=-\frac{\chi_{\perp}-\chi_{\|}}{2}[\boldsymbol{l} \mathbf{H}]^{2},
$$

where $\chi_{\|}\left(\chi_{\perp}\right)$ is the magnetic susceptibility parallel (perpendicular) to the vector $\boldsymbol{l}$. In collinear antiferromagnets, $\chi_{\|}<\chi_{\perp}$.

If the magnetic field $\mathbf{H}$ is oriented along the $y$ direction, the minimization of the sum of the energies [Eqs. (1) and (12)] gives $l_{x}^{2}-l_{y}^{2}=h^{2}$, for $H<H_{c}$, and $l_{y}=0$, for $H>$ $H_{c}$; here $h=H / H_{c}, H_{c}^{2}=2 \beta /\left(\chi_{\perp}-\chi_{\|}\right)$. First, the magnetic field leads to the appearance of homogeneous deviatoric stresses $\sigma_{x x}=-\sigma_{y y}=\gamma_{2}\left(l_{x}^{2}-l_{y}^{2}\right)$ due to the second term of the magnetoelastic energy [Eq. (2)]. These stresses are equal in the coexisting domains and do not affect the periodicity. The second effect is the renormalization of the equilibrium strain tensor [Eq. (4)] by the factor $\left(1-h^{4}\right)^{1 / 2}$. The third effect is the renormalization of the energy of the domain walls.

In order to find the domain wall structure, one should take into account the energy of the spin space inhomogeneous rotation

$$
E_{\mathrm{ex}}=\frac{g}{2}\left(\partial_{x} \varphi\right)^{2},
$$

where $g$ is a constant of the exchange interaction and $\varphi$ is the rotation angle of the unit antiferromagnetic vector $\left(l_{x}=\cos \varphi\right)$. The variation of the sum of energies [Eqs. (1), (12), and (13)] gives the equilibrium equation

$$
\delta^{2} \partial_{x}^{2} \varphi=\left(h^{2}+\sin ^{2} \varphi-\cos ^{2} \varphi\right) \sin \varphi \cos \varphi,
$$

where $\delta=(g / 2 \beta)^{1 / 2}$ is the effective thickness of the domain wall. The first integral of this equation is

$$
\left(\delta \partial_{x} \varphi\right)^{2}=\left(\frac{1-h^{2}}{2}-\sin ^{2} \varphi\right)^{2}
$$

The constant of integration is defined by the condition far from the domain wall where $\partial_{x} \varphi=0$.

Finally, we find the domain wall energy

$$
\sigma^{ \pm}(h)=\sigma_{0}\left(\sqrt{1-h^{4}} \pm 2 h^{2} \arcsin \sqrt{\frac{1 \pm h^{2}}{2}}\right),
$$

where $\sigma_{0}=\beta \delta$ is the energy of the domain wall without magnetic field. The minus sign in Eq. (16) corresponds to the domain walls presented in Fig. 3(a). These walls disappear at the critical field: $\rightarrow|\rightarrow| \rightarrow \mid \rightarrow \Rightarrow \rightarrow \longrightarrow \longrightarrow \longrightarrow$ because the difference between neighboring domains vanishes and the rotation in the wall tends to zero. The plus sign corresponds to the domain walls presented in Fig. 3(b). These walls remain at $h>1: \rightarrow|\leftarrow| \rightarrow \mid \leftarrow$. The possibility of such plain defects appearing in antiferromagnets was considered by Horovitz [8]. If the field is oriented along the $x$ direction, the behavior of the domain walls in Figs. 3(a) and 3(b) will be opposite. The energy of the walls of type ( $a$ ) will be $\sigma^{+}$, and at the critical field we obtain the following domain structures: $\uparrow|\downarrow| \uparrow \mid \downarrow$. The
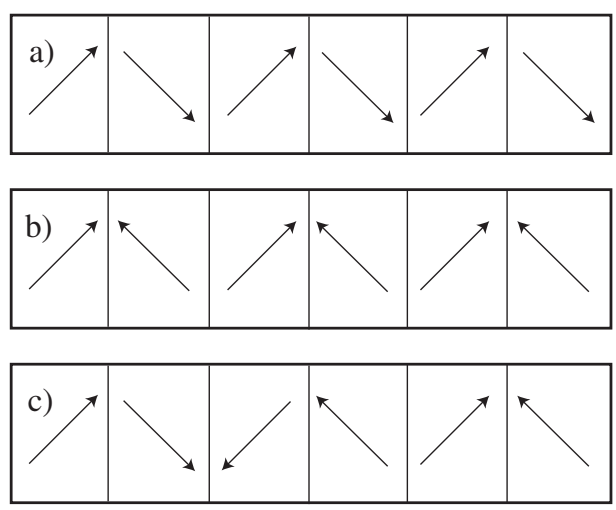

FIG. 3. (a) and (b) Two types of ordered domain structures and (c) a disordered domain structure.

walls of type $(b)$ will have energy $\sigma^{-}$and vanish at the critical field $\uparrow|\uparrow| \uparrow \mid \uparrow \Rightarrow \uparrow \uparrow \uparrow$.

In the general case, one starts from an arbitrary distribution of the types of walls, as is presented in Fig. 3(c). It leads to a complicated and even irreversible behavior of the periodicity for increasing and decreasing magnetic fields. Nonetheless, a simple behavior can be achieved if one starts by applying a field higher than $H_{c}$, say, in the $y$ direction. In this case, in equilibrium, one obtains a homogeneous state (say, $l_{x}=1$ ). By decreasing the field to $H<$ $H_{c}$, a domain structure will appear with energetically preferable walls, with the minus sign in Eq. (16) [11]. Then the field dependence of the period is given by

$$
d^{-}(h)=\frac{d_{0}}{\sqrt{1-h^{4}}}\left(\sqrt{1-h^{4}}-2 h^{2} \arcsin \sqrt{\frac{1-h^{2}}{2}}\right)^{1 / 2}
$$

see the lower curve in Fig. 4. This behavior will be reversible in the magnetic field. If then, after turning off the field, one applies a field in the $x$ direction, the domain walls will become unfavorable, and with increasing field the period will increase in accordance with

$$
d^{+}(h)=\frac{d_{0}}{\sqrt{1-h^{4}}}\left(\sqrt{1-h^{4}}+2 h^{2} \arcsin \sqrt{\frac{1+h^{2}}{2}}\right)^{1 / 2}
$$

see the upper curve in Fig. 4. But, if one stops the increase of the field at some value $H<H_{c}$ and starts to decrease it, the period will decrease more rapidly than the upper curve, because it is favorable to produce new walls with the minimal energy. Note that if magnetoelastic effects are comparable with the anisotropy [Eq. (1)], all obtained results for the behavior in the magnetic field remain valid. One merely should renormalize the value of $H_{c}$ and $\delta$.

The behavior of the domain structure will be much more complicated if one applies a field with some arbitrary orientation. Then the nearest-neighbor domains should have different widths. Let us calculate the concentrations of different domains $c^{+}$and $c^{-}\left(c^{+}+c^{-}=1\right)$, in the limit of small magnetic fields $H_{x}, H_{y} \ll H_{c}$ and, as before, 


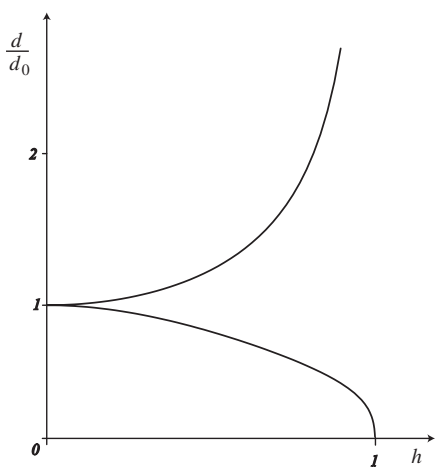

FIG. 4. Dependence of the period of the domain structure on the magnetic field.

assuming magnetoelastic effects to be small compared to the anisotropy. In this approximation, we can neglect the small rotation of the domain structure, which inevitably arises in the general case, and also set $l_{x}^{2}=l_{y}^{2} \approx 1 / 2$. Since the average strain is zero, we have $u_{y y}=0$ and $u_{x i}^{+} c^{+}+$ $u_{x i}^{-} c^{-}=0(i=x, y)$. At the domain wall, we have boundary conditions of mechanical equilibrium $\sigma_{x i}^{+}=\sigma_{x i}^{-}=\sigma_{x i}$ and phase equilibrium [12]

$$
F^{+}-\sigma_{x x} u_{x x}^{+}-2 \sigma_{x y} u_{x y}^{+}=F^{-}-\sigma_{x x} u_{x x}^{-}-2 \sigma_{x y} u_{x y}^{-},
$$

where $F=F_{\mathrm{me}}+F_{\mathrm{el}}+F_{H} ; \sigma_{x x}=\partial F / \partial u_{x x}$ and $\sigma_{x y}=$ $\frac{1}{2} \partial F / \partial u_{x y}$ are the components of the stress tensor. Solving this system of equations, we find the concentrations of the different domains:

$$
c^{ \pm}=\frac{1}{2}\left(1 \pm \frac{H_{x} H_{y}}{H_{\mathrm{me}}^{2}}\right), \quad H_{\mathrm{me}}^{2}=\frac{\gamma_{1}^{2}}{4 \mu_{1}\left(\chi_{\perp}-\chi_{\|}\right)} \ll H_{c}^{2} .
$$

The domain period cannot be found analytically at arbitrary field orientations. In this case, there are no symmetry arguments forcing the domain walls, near the samplesubstrate boundary (at distances of the order of the period), to be flat and oriented along the $z$ axis. The domain walls should be inclined and curved in this case.

Let us estimate the parameters of the theory. The anisotropy energy parameter is $\beta \sim \alpha^{4} U a^{-3}$, where $\alpha \sim 10^{-2}$ is the fine structure constant, $U \sim 10^{4} \mathrm{~K}$ is the atomic energy, and $a \sim 10^{-8} \mathrm{~cm}$ is the atomic size. The constant $g \sim J / a$, where $J \sim 10^{2} \mathrm{~K}$ is the exchange energy. Then, for the domain wall width, we find $\delta \sim(g / \beta)^{1 / 2} \sim a \alpha^{-2} \sqrt{J / U} \sim$ $10^{-5} \mathrm{~cm}$. The shear modulus of a typical material is $\mu \sim U a^{-3}$, and the magnetoelastic coupling constant $\gamma \sim$ $\alpha^{2} U a^{-3}$. Finally, for a crystal with thickness $L \sim 0.5 \mathrm{~mm}$, we obtain a reasonable estimation of the period $d \sim \sqrt{\delta L}$ to be of the order of a few microns.
Note that, for easy plane antiferromagnets with hexagonal crystal symmetry, anisotropy effects in the plane are small ( $\sim \alpha^{6}$ ) compared to magnetoelastic effects [Eq. (2)] $\left(\gamma_{1}=4 \gamma_{2}\right)$. If we neglect this anisotropy, the orientation of the domain structure will be arbitrary in the absence of a magnetic field. The antiferromagnetic vector will be oriented at an angle $\pm \pi / 4$ to the domain walls. In the presence of the magnetic field, the domain structure will presumably be oriented perpendicular to the field [11]. The critical field is $H_{c} \sim \gamma / \sqrt{\mu\left(\chi_{\perp}-\chi_{\|}\right)}$in this case. The domain concentration remains $1 / 2$, and the dependence of the period on the magnetic field will be described by the value of $d(h)$ obtained in the tetragonal case.

Finally, we note that the paper by Horovitz [8] deals with the transition in the antiferromagnetic polarization due to a microscopic mechanism: condensation of the holes into a charge-density wave. In our description, all microscopic details, leading to a specific antiferromagnetic polarization without magnetic field, are hidden in a few parameters (anisotropy $\beta$ and constants of magnetoelastic interaction $\gamma)$. Then we calculate the dependence of the equilibrium period and concentrations of different domains on an external magnetic field.

This work is supported in part by the RFBR Grant No. 06-02-17281 and by the CRDF Grant No. RUE11625-MO-06. V.I. M. thanks Forschungszentrum Jülich for its hospitality.

[1] A. Janossy et al., Phys. Rev. B 59, 1176 (1999).

[2] A. Janossy, T. Feher, and A. Erb, Phys. Rev. Lett. 91, 177001 (2003).

[3] L. Ya. Vinnikov et al., JETP Lett. 77, 502 (2003).

[4] L. Ya. Vinnikov, I. V. Grigor'eva, and L. A. Gurevich, Springer Ser. Mater. Sci. 23, 89 (1993).

[5] A. F. Andreev and V. I. Marchenko, Sov. Phys. Usp. 23, 21 (1980).

[6] A. G. Khachaturyan, Theory of Structural Transformation in Solids (Wiley, New York, 1983).

[7] B. Horovitz, G. R. Barsch, and J.A. Krumhansl, Phys. Rev. B 43, 1021 (1991).

[8] B. Horovitz, Phys. Rev. B 69, 140501(R) (2004).

[9] E. Gomonay and V. M. Loktev, J. Phys. Condens. Matter 14, 3959 (2002); Phys. Solid State 47, 1755 (2005).

[10] V. M. Kalita, A. F. Lozenko, S. M. Ryabchenko, and P. A. Trotsenko, Zh. Eksp. Teor. Fiz. 126, 1209 (2004) [JETP 99, 1054 (2004)].

[11] The global domain wall orientations ( $x$ or $y$ ) remain degenerate in the considered approximation. However, if we take into account demagnetization effects, the orientation of the domain structure with walls normal to the applied field will be more favorable.

[12] I. A. Privorotskii, Zh. Eksp. Teor. Fiz. 60, 1525 (1971) [Sov. Phys. JETP 33, 825 (1971)]. In Eq. (17) we have neglected demagnetization effects $(\chi \ll 1)$. 